\title{
Sampling with Bessel Functions
}

\author{
K.I. Kou, T. Qian and F. Sommen
}

\begin{abstract}
The paper deals with sampling of $\sigma$-bandlimited functions in $\mathbf{R}^{m}$ with Clifford-valued, where bandlimitedness means that the spectrum is contained in the ball $B(0, \sigma)$ that is centered at the origin and has radius $\sigma$. By comparing with the general setting, what is new in the sampling is using the explicit Bochner-type relations involving spherical harmonics and monogenics in the Clifford algebra setting. Convergence of the sampling formulas in the $L^{2}$ sense and in the uniform and absolute pointwise sense are studied.
\end{abstract}

Mathematics Subject Classification (2000). Primary 42A38; Secondary 30 G35. Keywords. sampling, spherical harmonics, spherical monogenics, Bessel functions.

\section{Introduction}

The Bessel function of arbitrary complex order $\nu$ is defined by ([16] 3.1(8))

$$
J_{\nu}(z)=\sum_{k=0}^{\infty} \frac{(-1)^{k}(z / 2)^{\nu+2 k}}{k ! \Gamma(k+\nu+1)},
$$

where $1 / \Gamma(k+\nu+1)$ are assigned to be of zero value for non-positive integers $k+\nu+1$ due to analytic continuation of the function $1 / \Gamma(z)$. It is shown that for all $\nu$ the Bessel function defined through (1.1) satisfies the corresponding Bessel's differential equation $([16] 3.1(1))$

$$
z^{2} \frac{d^{2} y}{d z^{2}}+z \frac{d y}{d z}+\left(z^{2}-\nu^{2}\right) y=0
$$

For complex numbers $\nu$ with $\operatorname{Re} \nu>-\frac{1}{2}$, there holds the integral formula ([16] 3.3 (1))

$$
J_{\nu}(z)=\frac{(z / 2)^{\nu}}{\sqrt{\pi} \Gamma(\nu+1 / 2)} \int_{0}^{\pi} \cos (z \cos \theta) \sin ^{2 \nu} \theta d \theta
$$

The study was supported by Research Grant of the University of Macau No. RG059/0506S/QT/FST. 
Bessel's equation arises when finding separable solutions to Laplace's equation and the Helmholtz equation in cylindrical or spherical coordinates, and Bessel functions are therefore especially important for many problems of wave propagation, static potentials, and so on. For cylindrical problems, one obtains Bessel functions of integer order $\nu=n$; for spherical problems, one obtains half integer orders $\nu=n+\frac{1}{2}$, including electromagnetic waves in a cylindrical waveguide; heat conduction in a cylindrical object and modes of vibration of a thin circular (or annular) membrane. Bessel functions also have useful properties for other problems, such as signal processing (e.g., Frequency modulation synthesis and Kaiser window), see [16] and [2].

The function $J_{\nu}(z)$ has a countably infinite number of positive real zeros, and a finite number of conjugate complex zeros. Moreover, all the zeros are simple, except possibly the zero at the point $z=0$ ([14] 5.13 Theorem 2). Denote these real zeros in the ascending order $0<u_{1}^{(\nu)}<u_{2}^{(\nu)}<\cdots<u_{j}^{(\nu)}<\cdots$, where $u_{j}^{(\nu)}$ is called the $j$ th positive zero of $J_{\nu}$.

To treat a bandlimited function (see Definition 1.1) we will encounter a positive number $\sigma$ as, or larger than the spectrum radius. Throughout the following discussion we will deal with an arbitrary but fixed positive parameter $\sigma$. To simplify the notation, we let

$$
\lambda_{j}^{(\nu)}=\frac{u_{j}^{(\nu)}}{\sigma}, \quad j=1,2,3, \ldots
$$

So $\lambda_{j}^{(\nu)}$ is the value of the $j$ th positive zero of $J_{\nu}$ scaled by a fixed factor $1 / \sigma$.

Accordingly, we have the orthogonal property

$$
\int_{0}^{\sigma} J_{\nu}\left(s \lambda_{j}^{(\nu)}\right) J_{\nu}\left(s \lambda_{k}^{(\nu)}\right) s d s=\frac{\sigma^{2} \delta_{j, k}}{2} J_{\nu+1}^{2}\left(u_{j}^{(\nu)}\right),
$$

where Re $\nu>-1, \delta_{j, k}$ is the Kronecker delta ([14] 5.14 (5.14.4), (5.14.6)).

If $F$ is square-integrable with respect to the weight $s d s$ on $(0, \sigma)$, then $F$ has a Bessel series expansion of order $\nu$ on the interval $(0, \sigma)$ given by

$$
F(s)=\sum_{j=1}^{\infty} A_{j} J_{\nu}\left(s \lambda_{j}^{(\nu)}\right)
$$

where $A_{j}$ can be determined by the orthogonality property of $J_{\nu}$ in (1.3)

$$
A_{j}=\frac{2}{\sigma^{2} J_{\nu+1}^{2}\left(u_{j}^{(\nu)}\right)} \int_{0}^{\sigma} F(s) J_{\nu}\left(s \lambda_{j}^{(\nu)}\right) s d s .
$$


The number $A_{j}$ is called the $j$ th Bessel coefficient of the function $F$ ([14] 5.14 $(5.14 .1),(5.14 .7))$.

Parseval's identity for Bessel series (1.4) reads

$$
\int_{0}^{\sigma}|F(s)|^{2} s d s=\|F\|^{2}=\sum_{j=1}^{\infty} \frac{\sigma^{2}}{2} J_{\nu+1}^{2}\left(u_{j}^{(\nu)}\right) A_{j}^{2} .
$$

Fourier transform of functions in $L^{1}\left(\mathbf{R}^{m}\right)$ is defined by

$$
\widehat{f}(\underline{\xi})=\int_{\mathbf{R}^{m}} e^{-\mathbf{i}<\underline{x}, \underline{\xi}>} f(\underline{x}) d \underline{x} .
$$

The inverse Fourier transform is formally defined by

$$
\check{g}(\underline{x})=\frac{1}{(2 \pi)^{m}} \int_{\mathbf{R}^{m}} e^{\mathbf{i}<\underline{x}, \underline{\xi}>} g(\underline{\xi}) d \underline{\xi} .
$$

We now define a class of functions in terms of their Fourier transforms.

Definition 1.1. If $f \in L^{2}\left(\mathbf{R}^{m}\right)$ and supp $\widehat{f} \subset B(0, \sigma)$ (the ball in $\mathbf{R}^{m}$ centered at 0 with radius $\sigma$ ), then $f$ is called a bandlimited function with bandwidth $\sigma$.

The paper is organized as follows. In the second section we review the general sampling theory. In section 3 by using the decomposition of square integrable functions into spherical harmonics, we formulate a corresponding sampling theorem. In section 4 we establish the sampling result in relation to homogeneous left-monogenic functions.

The symbols $\mathbf{Z}$ and $\mathbf{N}$ denote the sets of the integers and the natural numbers, respectively.

\section{The General Theory of Sampling Theorems}

We will be working with the weighted $L^{2}$ space $L^{2}(I, \rho)(I \subset \mathbf{R}$ and $\rho(s) \geq 0$ a.e. in $I$ ). The inner product is given by

$$
<F, G>=\int_{I} F(s) \overline{G(s)} \rho(s) d s .
$$

The norm of $F$ with respect to the weighted Lebesgue measure is

$$
\|F\|=<F, F>^{1 / 2}=\left(\int_{I}|F(s)|^{2} \rho(s) d s\right)^{1 / 2} .
$$

In the rest of the note we will fix the weight function $\rho(s)=s$.

Our question of sampling and reconstruction can be stated as follows: For a set $R$ of functions on a domain $\Omega$, can we identify a discrete set $\left\{r_{j}\right\} \in \Omega$ such 
that every $f \in R$ is uniquely determined by its values on $\left\{r_{j}\right\}$ ? If such a discrete set $\left\{r_{j}\right\}$ exists, we, specifically, expect to have a sampling formula of the form

$$
f(r)=\sum_{j} f\left(r_{j}\right) S_{j}(r)
$$

valid for all functions $f$ in $R$ where $S_{j}$ are some known functions, and the convergence is in a certain norm or point-wise sense.

Sampling in Bessel functions fits into the following frame work but with some variations.

Assume that we have an integral kernel $K(\cdot, r) \in L^{2}(I, \rho)$, and there exists a sequence $\left\{r_{j}\right\}_{j=1}^{\infty} \in \Omega$ such that $K\left(s, r_{j}\right)=a_{j} \overline{\phi_{j}}(s)$, where $a_{j}>0$ are normalizing constants so that $\left\{\phi_{j}\right\}_{j=1}^{\infty}$ forms an orthonormal basis for $L^{2}(I, \rho)$.

Now define on $L^{2}(I, \rho)$ the linear integral transformation with the kernel $K(s, r)$ mapping $F \in L^{2}(I, \rho)$ to

$$
f(r):=\int_{I} F(s) K(s, r) \rho(s) d s .
$$

The integral transformation (2.2) is well defined because $F$ and $K(\cdot, r)$ both are assumed to be in $L^{2}(I, \rho)$. Since $\left\{\phi_{j}\right\}_{j=1}^{\infty}$ is a basis the transformation is one to one.

Next we expand the function $K(s, r)$ into

$$
K(s, r)=\sum_{j=1}^{\infty} S_{j}(r) \overline{\phi_{j}}(s), \quad(s, r) \in I \times \Omega,
$$

where

$$
S_{j}(r)=<K(\cdot, r), \overline{\phi_{j}}(\cdot)>.
$$

The inner product is one associated with the Hilbert space $L^{2}(I, \rho)$. Substituting (2.3) into (2.2) and interchanging the order of summation and integration due to the $L^{2}$-convergence, we obtain

$$
f(r)=\sum_{j=1}^{\infty} S_{j}(r) \int_{I} F(s) \overline{\phi_{j}}(s) \rho(s) d s=\sum_{j=1}^{\infty} S_{j}(r)<F, \phi_{j}>.
$$

Recalling the relation $\overline{\phi_{j}}(s)=\frac{K\left(s, r_{j}\right)}{a_{j}}$, and $(2.2)$, we have $<F, \phi_{j}>=\frac{f\left(r_{j}\right)}{a_{j}}$ and

$$
f(r)=\sum_{j=1}^{\infty} \frac{f\left(r_{j}\right)}{a_{j}} S_{j}(r) .
$$


This holds in the point-wise convergence sense for all functions $f$ in the range of the integral transformation (2.2), viz.

$$
R=\left\{f: \Omega \rightarrow \mathbf{C} \text { such that } f(r)=\int_{I} F(s) K(s, r) \rho(s) d s, F \in L^{2}(I, \rho)\right\} .
$$

To study uniform convergence, we first have

$$
\begin{aligned}
\left|f(r)-f^{(N)}(r)\right| & \leq \sum_{j=N+1}^{\infty}\left|\frac{f\left(r_{j}\right)}{a_{j}} S_{j}(r)\right| \\
& \leq\left(\sum_{j=N+1}^{\infty} \frac{\left|f\left(r_{j}\right)\right|^{2}}{a_{j}^{2}}\right)^{1 / 2}\left(\sum_{j=N+1}^{\infty}\left|S_{j}(r)\right|^{2}\right)^{1 / 2}
\end{aligned}
$$

where $f^{(N)}(r)=\sum_{j=1}^{N} \frac{f\left(r_{j}\right)}{a_{j}} S_{j}(r)$. In the expansion

$$
F(s)=\sum_{j=1}^{\infty} A_{j} \phi_{j}(s)
$$

the coefficients

$$
A_{j}=<F, \phi_{j}>=\frac{f\left(r_{j}\right)}{a_{j}} .
$$

The Plancherel identity gives

$$
\|F\|^{2}=\sum_{j=1}^{\infty} \frac{\left|f\left(r_{j}\right)\right|^{2}}{a_{j}^{2}}<\infty
$$

So, if $N$ is large enough, then the tail

$$
\sum_{j=N+1}^{\infty} \frac{\left|f\left(r_{j}\right)\right|^{2}}{a_{j}^{2}}
$$

is small. Based on this, if there exists $M<\infty$ and $B \subset \Omega$ such that for large enough $N$

$$
\sum_{j=N+1}^{\infty}\left|S_{j}(r)\right|^{2}<M, \quad r \in B
$$

then the point-wise convergence in (2.5) is strengthened to becomes uniform and absolute in $B$.

Note that the condition

$$
\|K(\cdot, r)\|^{2}=\sum_{j=1}^{\infty}\left|S_{j}(r)\right|^{2} \leq M, \quad r \in B,
$$

implies (2.7). 
Now we establish $L^{2}$ convergence of (2.5). Based on the injective mapping (2.2) we may equip a Hilbert space structure onto the set $R$ by letting $\|f\|_{H}=\|F\|$, where $H$ denotes the formulated Hilbert space. The convergence of (2.5) then may be seen to be also in the norm $\|\cdot\|_{H}$. In fact, due to the polarization identity, the transformation (2.2) under the defined norm induces an isometrical isomorphism from $H$ to $L^{2}(I, \rho)$. Under the isomorphism orthonormal bases in $L^{2}(I, \rho)$ are mapped to orthonormal bases in $H$. Since $\left\{\phi_{i}\right\}_{i=1}^{\infty}$ is an orthonormal basis in $L^{2}(I, \rho)$, then the relation (2.4) exhibits that $\left\{S_{j}\right\}_{j=1}^{\infty}$ is an orthonormal basis in $H$. This concludes that the convergence of $(2.5)$ is in the norm $\|\cdot\|_{H}$ sense.

For more comprehensive explanations of this general sampling theory, the reader may refer to [5], [6], [9], [12] and [13].The underlying idea of the procedure is borrowed from Hardy [11] who first noticed that sampling formula (2.1) is an orthogonal expansion.

\section{Sampling in relation to decomposition into spherical harmonics}

The work will be based on the direct sum decomposition

$$
L^{2}\left(\mathbf{R}^{m}\right)=\sum_{k=0}^{\infty} \bigoplus N_{k}
$$

where the dimension $m \geq 2, N_{k}(k \geq 0)$ is the set of finite linear combinations of scalar valued functions of the form $g(r) Y_{k}(\underline{x})$, where $r=|\underline{x}|, g$ is a function defined on $[0, \infty)$ satisfying $\int_{0}^{\infty}|g(r)|^{2} r^{m+2 k-1} d r<\infty$ and $Y_{k}$ a solid spherical harmonics of degree $k$. Each space $N_{k}$ is invariant under Fourier transformation ([15] p.151).

The space of solid spherical harmonics of degree $k$, being isomorphic to the space of surface spherical harmonics of degree $k$, denoted by $Y_{k}$, has finite dimension $\gamma_{k}=\frac{(m+k-1) !}{k !(m-1) !}$. When $k=0$, the space $N_{0}$ is of dimension 1 generated by the constant function 1 , and functions $g(r) Y_{0}(\underline{x})$ are reduced to just radial functions $g(r)$. The following theorem gives the sampling expansion for bandlimited functions in $N_{0}$.

Theorem 3.1. Let $f$ be a radial function $f(\underline{x}):=f_{0}(r)(r=|\underline{x}|)$ in $L^{2}\left(\mathbf{R}^{m}\right)$ and $f$ be bandlimited with bandwidth $\sigma$. Then for all $\underline{x} \in \mathbf{R}^{m}$ we have

$$
f(\underline{x})=\sum_{j=1}^{\infty} f_{0}\left(\lambda_{j}^{(c)}\right)\left(\frac{\lambda_{j}^{(c)}}{r}\right)^{c} S_{c ; \lambda_{j}^{(c)}}(r)
$$


where

$$
S_{c ; \lambda_{j}^{(c)}}(r)= \begin{cases}\frac{2 \lambda_{j}^{(c)} J_{c}(\sigma r)}{\sigma J_{c+1}\left(\sigma \lambda_{j}^{(c)}\right)\left(\lambda_{j}^{(c)^{2}}-r^{2}\right)}, & r \neq \lambda_{j}^{(c)} \\ 1, & r=\lambda_{j}^{(c)},\end{cases}
$$

and $c=\frac{m-2}{2}, \lambda_{j}^{(c)}$ are the scaled positive zeros of the Bessel function $J_{c}$ given by (1.2), and the series (3.2) being convergent in $L^{2}$-norm, and absolutely, uniformly, point-wisely convergent on the whole space $\mathbf{R}^{m}$.

Proof. The Fourier transform of a radial function is still radial. With an abuse of notation but informatively, we write $\widehat{f}(\underline{t}):=\widehat{f}_{0}(s)(s=|\underline{t}|)$ for all $\underline{t} \in \mathbf{R}^{m}$. By the assumption $f \in L^{2}\left(\mathbf{R}^{m}\right)$, being bandlimited with bandwidth $\sigma$, the inverse Fourier formula ([15] Theorem 3.3 in Chapter 4) gives

$$
f_{0}(r)=(2 \pi)^{-\frac{m}{2}} \int_{0}^{\sigma}\left[\widehat{f}_{0}(s) s^{\frac{m-2}{2}}\right]\left[r^{-\frac{m-2}{2}} J_{\frac{m-2}{2}}(r s)\right] s d s .
$$

This corresponds to the integral transformation (2.2) with $I=[0, \sigma], \rho(s)=s$, $c=\frac{m-2}{2}, F(s)=(2 \pi)^{-\frac{m}{2}} \widehat{f}_{0}(s) s^{c}$ and the integral kernel $K(s, r)=r^{-c} J_{c}(r s)$.

Recalling (1.3), the set of functions

$$
\overline{\phi_{j}}(s)=\frac{J_{c}\left(s \lambda_{j}^{(c)}\right)}{\left\|J_{c}\left(\cdot \lambda_{j}^{(c)}\right)\right\|}=\frac{\sqrt{2} J_{c}\left(s \lambda_{j}^{(c)}\right)}{\sigma J_{c+1}\left(u_{j}^{(c)}\right)}
$$

forms an orthonormal basis for $L^{2}(I, \rho)$. For $r_{j}=\lambda_{j}^{(c)}$ we have $K\left(s, r_{j}\right)=a_{j} \overline{\phi_{j}}(s)$ with

$$
a_{j}=\frac{\left\|J_{c}\left(\cdot \lambda_{j}^{(c)}\right)\right\|}{\left(\lambda_{j}^{(c)}\right)^{c}}=\frac{\sigma J_{c+1}\left(u_{j}^{(c)}\right)}{\sqrt{2}\left(\lambda_{j}^{(c)}\right)^{c}} .
$$

Next expand $K(s, r)$ into $(2.3)$ with $\overline{\phi_{j}}(s)$ given in (3.5), as Bessel series expansion of order $c=\frac{m-2}{2}$, we have

$$
r^{-c} J_{c}(r s)=\sum_{j=1}^{\infty} r^{-c} S_{c ; \lambda_{j}^{(c)}}(r) J_{c}\left(s \lambda_{j}^{(c)}\right) .
$$

Using special function formula (see [1], 11.3.29), for order $c$ we have

$$
S_{c ; \lambda_{j}^{(c)}}(r)= \begin{cases}\frac{2 \lambda_{j}^{(c)} J_{c}(\sigma r)}{\sigma J_{c+1}\left(\sigma \lambda_{j}^{(c)}\right)\left(\lambda_{j}^{(c)}{ }^{2}-r^{2}\right)}, & r \neq \lambda_{j}^{(c)} ; \\ 1, & r=\lambda_{j}^{(c)} .\end{cases}
$$

Hence the corresponding coefficients in (2.3) are

$$
S_{j}(r)=\left\|J_{c}\left(\cdot \lambda_{j}^{(c)}\right)\right\| r^{-c} S_{c ; \lambda_{j}^{(c)}}(r)=\frac{\sigma J_{c+1}\left(u_{j}^{(c)}\right)}{\sqrt{2} r^{c}} S_{c ; \lambda_{j}^{(c)}}(r) .
$$


Substituting (3.6) into (3.4), we have

$$
\begin{aligned}
f(\underline{x}) & =(2 \pi)^{-\frac{m}{2}} r^{-c} \int_{0}^{\sigma} \widehat{f}_{0}(s) \sum_{j=1}^{\infty} S_{c ; \lambda_{j}^{(c)}}(r) J_{c}\left(s \lambda_{j}^{(c)}\right) s^{c+1} d s \\
& =r^{-c} \sum_{j=1}^{\infty} S_{c ; \lambda_{j}^{(c)}}(r)\left[(2 \pi)^{-\frac{m}{2}} \int_{0}^{\sigma} \widehat{f}_{0}(s) J_{c}\left(s \lambda_{j}^{(c)}\right) s^{c+1} d s\right],
\end{aligned}
$$

where interchanging the order of summation and integration is due to the $L^{2}$ convergence. Using (3.4),

$$
(2 \pi)^{-\frac{m}{2}} \int_{0}^{\sigma} \widehat{f}_{0}(s) J_{c}\left(s \lambda_{j}^{(c)}\right) s^{c+1} d s=\left(\lambda_{j}^{(c)}\right)^{c} f_{0}\left(\lambda_{j}^{(c)}\right) .
$$

This, together with (3.8), gives (3.2).

Now we study uniform convergence. To this end, based on the general theory, it is sufficient to estimate

$$
\|K(\cdot, r)\|^{2}=\left\|r^{-c} J_{c}(r(\cdot))\right\|^{2}=r^{-2 c} \int_{0}^{\sigma} J_{c}^{2}(r s) s d s .
$$

using the modified expressions in ([3] p.102), for Re $\nu>-1$, we have

$$
\begin{aligned}
& \int_{0}^{\sigma} J_{\nu}\left(\frac{\gamma}{\sigma} s\right) J_{\nu}\left(\frac{\beta}{\sigma} s\right) s d s \\
= & \begin{cases}\frac{\sigma^{2} \gamma \beta}{2 \nu\left(\beta^{2}-\gamma^{2}\right)}\left[J_{\nu-1}(\gamma) J_{\nu+1}(\beta)-J_{\nu+1}(\gamma) J_{\nu-1}(\beta)\right], & \gamma \neq \beta ; \\
\frac{\sigma^{2}}{2}\left[J_{\nu}^{2}(\gamma)-J_{\nu-1}(\gamma) J_{\nu+1}(\gamma)\right], & \gamma=\beta .\end{cases}
\end{aligned}
$$

For $\gamma=\beta=r \sigma$ and $\nu=c=\frac{m-2}{2} \geq 0$,

$$
r^{-2 c} \int_{0}^{\sigma} J_{c}^{2}(r s) s d s=\frac{\sigma^{2}}{2}\left[\frac{J_{c}^{2}(r \sigma)}{r^{2 c}}-\frac{J_{c-1}(r \sigma) J_{c+1}(r \sigma)}{r^{2 c}}\right] .
$$

The estimate of the bounds of (3.10) for the cases $m>3$ is based on the following asymptotic results. For the Bessel functions of order $\operatorname{Re} \nu>0$ there holds

$$
J_{\nu}(r) \approx \frac{r^{\nu}}{2^{\nu} \Gamma(1+\nu)}
$$

while for large arguments $r>>1$,

$$
J_{\nu}(r) \approx \sqrt{\frac{2}{\pi r}} \cos \left(r-\frac{1}{2} \nu \pi-\frac{1}{4} \pi\right)
$$

$([14](5.16 .1))$.

For $m=2, J_{\frac{m-4}{2}}=J_{-1}(r)=-J_{1}(r)$ because of the property $J_{-m}(r)=$ $(-1)^{m} J_{m}(r)(m \in \mathbf{Z})([16] 2.1(2))$. For $m=3, J_{\frac{m-4}{2}}=J_{-\frac{1}{2}}(r)=\left(\frac{2}{\pi r}\right)^{1 / 2} \cos (r)$ 
([16] $3.4(6))$.

So the integral in (3.10) is bounded for $m \geq 2$ by a positive number $M$. This implies $\|K(\cdot, r)\|^{2}<M$ for all $r \in B=[0, \infty)$. Hence the series in (3.2) is uniformly convergent in the whole space.

Now we show the $L^{2}$-norm convergence of (3.2). Based on the general theory, by comparing (3.4) with (2.2), it is sufficient to prove that the norm $\|\cdot\|_{H}$ of $f_{0}$ defined through the relation

$$
\left\|f_{0}\right\|_{H}=\|F\|
$$

is a constant multiple of the norm $\|f\|_{L^{2}\left(\mathbf{R}^{m}\right)}$. This is to show that, for a constant $c$, we have

$$
\|f\|_{L^{2}\left(\mathbf{R}^{n}\right)}=c\|F\| .
$$

But this is just the Plancherel theorem of a radial square-integral function in $\mathbf{R}^{m}$.

This theorem shows that bandlimited radial functions can be constructed completely from the specified sampling. We now turn our attention to bandlimited square integrable functions not necessarily be radial. We begin by first examining the sampling formulas for functions in functions classes $N_{k}, k \geq 1$.

Theorem 3.2. Let $k$ be a fixed non-negative integer and $f$ be of the form $f(x):=$ $f_{0}(r) Y_{k}(\underline{x}) \quad(r=|\underline{x}|), Y_{k}$ a solid harmonics of degree $k$, in $L^{2}\left(\mathbf{R}^{m}\right)$, and $f_{0}$ be bandlimited with bandwidth $\sigma$. Then $\widehat{f}(\underline{t})=\widehat{f}_{0}(s) Y_{k}(\underline{t})(s=|\underline{t}|)$ with

$$
\widehat{f}_{0}(s)=i^{-k}(2 \pi)^{\frac{m}{2}} \int_{0}^{\infty} f_{0}(r) s^{-c_{k}} J_{c_{k}}(s r) r^{c_{k}+1} d r .
$$

and

$$
f_{0}(r)=i^{-k}(2 \pi)^{-\frac{m}{2}} \int_{0}^{\sigma} \widehat{f}_{0}(s) r^{-c_{k}} J_{c_{k}}(r s) s^{c_{k}+1} d s .
$$

Moreover

$$
f(\underline{x})=\sum_{j=1}^{\infty} f_{0}\left(\lambda_{j}^{\left(c_{k}\right)}\right)\left(\frac{\lambda_{j}^{\left(c_{k}\right)}}{r}\right)^{c_{k}} S_{c_{k} ; \lambda_{j}^{\left(c_{k}\right)}}(r) Y_{k}(\underline{x}),
$$

where $c_{k}=\frac{m+2 k-2}{2}, S_{c_{k} ; \lambda_{j}^{\left(c_{k}\right)}}$ is given by (3.3), $\lambda_{j}^{\left(c_{k}\right)}$ are the scaled positive zeros of the Bessel function $J_{c_{k}}$ given by (1.2), and the series (3.14) being convergent in $L^{2}$-norm, absolutely and uniformly convergent point-wisely on whole space $\mathbf{R}^{m}$.

Proof. The Fourier transform of the function $f(\underline{x}):=f_{0}(r) Y_{k}(\underline{x})(r=|\underline{x}|)$ can be expressed as $\widehat{f}(\underline{t})=\widehat{f}_{0}(s) Y_{k}(\underline{t})(s=|\underline{t}|)$, where $Y_{k}$ is a solid harmonics of degree $k$ ([15] Theorem 3.10) and,

$$
\widehat{f}_{0}(s)=i^{-k}(2 \pi)^{\frac{m}{2}} \int_{0}^{\infty}\left[f_{0}(r) r^{\frac{m+2 k-2}{2}}\right]\left[s^{-\frac{m+2 k-2}{2}} J_{\frac{m+2 k-2}{2}}(s r)\right] r d r .
$$


With supp $\widehat{f}_{0} \subset[0, \sigma]$ the radial function $f_{0}$ can be obtained via the formula

$$
f_{0}(r)=i^{-k}(2 \pi)^{-\frac{m}{2}} \int_{0}^{\sigma}\left[\widehat{f}_{0}(s) s^{\frac{m+2 k-2}{2}}\right]\left[r^{-\frac{m+2 k-2}{2}} J_{\frac{m+2 k-2}{2}}(r s)\right] s d s .
$$

Denote $c_{k}=\frac{m+2 k-2}{2}$. The integral transformation (3.15) maps $F(s)=i^{-k}(2 \pi)^{-\frac{m}{2}} \widehat{f}_{0}(s) s^{c_{k}}$ to $f_{0}(r)$, where the corresponding integral kernel is $K(s, r)=r^{-c_{k}} J_{c_{k}}(r s)$.

Also based on (1.3), the set of functions $K\left(s, \lambda_{j}^{\left(c_{k}\right)}\right)=a_{j} \overline{\phi_{j}}(s)$ gives rise to an orthonormal basis $\left\{\phi_{j}\right\}_{j=1}^{\infty}$ of $L^{2}(I, \rho)$, where

$$
a_{j}=\frac{\left\|J_{c_{k}}\left(\cdot \lambda_{j}^{\left(c_{k}\right)}\right)\right\|}{\left(\lambda_{j}^{\left(c_{k}\right)}\right)^{c_{k}}}=\frac{\sigma J_{c_{k}+1}\left(u_{j}^{\left(c_{k}\right)}\right)}{\sqrt{2}\left(\lambda_{j}^{\left(c_{k}\right)}\right)^{c_{k}}}
$$

and

$$
\overline{\phi_{j}}(s)=\frac{J_{c_{k}}\left(s \lambda_{j}^{\left(c_{k}\right)}\right)}{\left\|J_{c_{k}}\left(\cdot \lambda_{j}^{\left(c_{k}\right)}\right)\right\|}=\frac{\sqrt{2} J_{c_{k}}\left(s \lambda_{j}^{\left(c_{k}\right)}\right)}{\sigma J_{c_{k}+1}\left(u_{j}^{\left(c_{k}\right)}\right)} .
$$

The expansion of the function $K(s, r)$ into the series in $\overline{\phi_{j}}(s)$ is

$$
r^{-c_{k}} J_{c_{k}}(s r)=\sum_{j=1}^{\infty} r^{-c_{k}} S_{c_{k} ; \lambda_{j}^{\left(c_{k}\right)}}(r) J_{c_{k}}\left(s \lambda_{j}^{\left(c_{k}\right)}\right),
$$

where $S_{c_{k} ; \lambda_{j}^{\left(c_{k}\right)}}$ is given by (3.3). The corresponding coefficient

$$
S_{j}(r)=\frac{\sigma J_{c_{k}+1}\left(u_{j}^{\left(c_{k}\right)}\right)}{\sqrt{2} r^{c_{k}}} S_{c_{k} ; \lambda_{j}^{\left(c_{k}\right)}}(r)
$$

The proof of the point-wise convergence of (3.14) now follows from the general theory in Section 2. The absolute, uniform and point-wise convergence in $\mathbf{R}^{m}$ follows from the relation

$$
\|K(\cdot, r)\|^{2}=r^{-2 c_{k}} \int_{0}^{\sigma} J_{c_{k}}^{2}(r s) s d s,
$$

using formula (3.10) $\left(\gamma=\beta=r \sigma\right.$ and $\left.\nu=c_{k} \geq 0\right)$, and

$$
\int_{0}^{\sigma} J_{c_{k}}^{2}(r s) s d s=\frac{1}{2}\left[J_{c_{k}}^{2}(r \sigma)-J_{c_{k}-1}(r \sigma) J_{c_{k}+1}(r \sigma)\right] .
$$

This is nothing but an increased dimension to $m+2 k$. The same reasoning as in the proof of Theorem 3.1 based on the asymptotic formulas gives $\|K(\cdot, r)\|^{2}<M$ for all $r \in B=[0, \infty)$. Hence the series in (3.14) is absolutely and uniformly convergent in the whole space. 
To show the $L^{2}$-convergence of (3.14) we only need to point out that the $L^{2}\left(\mathbf{R}^{m}\right)$ norm of $f$ is a constant multiple of the Hilbert space norm of $f_{0}$ given by the relation

$$
\left\|f_{0}\right\|_{H}=\|F\|
$$

We finally remark that the mentioned constant depends only on the space dimension $m$ but not on $k$. This fact will be used in the proof of Theorem 3.3.

Let $\left\{Y_{k}^{(1)}, Y_{k}^{(2)}, \cdots, Y_{k}^{\left(\gamma_{k}\right)}\right\}$ be an orthonormal basis of $Y_{k}$, the space of surface spherical harmonics of degree $k$ in $\mathbf{R}^{m}$. For any $f_{k} \in N_{k}$,

$$
f_{k}(\underline{x})=\sum_{l=1}^{\gamma_{k}} f_{l}^{(k)}(r) r^{k} Y_{k}^{(l)}\left(\underline{x}^{\prime}\right),
$$

where $r=|\underline{x}|, \underline{x}=r \underline{x}^{\prime}$ and $f_{l}^{(k)}$ are functions defined on $[0, \infty), l=1,2, \cdots, \gamma_{k}$, satisfying $\int_{0}^{\infty}\left|f_{l}^{(k)}(r)\right|^{2} r^{m+2 k-1} d r<\infty$.

If $f$ and $g \in Y_{k}$, the inner product in $L^{2}\left(\Sigma_{m-1}\right)$ is defined by

$$
(f, g)=\int_{\Sigma_{m-1}} f\left(\underline{x}^{\prime}\right) \overline{g\left(\underline{x}^{\prime}\right)} d \underline{x}^{\prime},
$$

where $\Sigma_{m-1}$ is the $(m-1)$-dimensional unit sphere in $\mathbf{R}^{m}$ and $d \underline{x}$ is the $(m-1)$ dimensional Lebesgue area measure on $\Sigma_{m-1}$. The sampling formula of $f_{k} \in N_{k}$ is a summation of $\gamma_{k}$ formulas of the ones obtained in Theorem 3.2.

Next we derive the sampling expansions of square integrable bandlimited functions in $\mathbf{R}^{m}$.

Theorem 3.3. Let $f \in L^{2}\left(\mathbf{R}^{m}\right)$ be bandlimited with bandwidth $\sigma$. Then

$$
f(\underline{x})=\sum_{k=0}^{\infty} f_{k}(\underline{x})=\sum_{k=0}^{\infty} \sum_{l=1}^{\gamma_{k}} f_{l}^{(k)}(r) Y_{k}^{(l)}(\underline{x})
$$

where $r=|\underline{x}|$, and $f$ can be reconstructed completely via

$$
f(\underline{x})=\sum_{k=0}^{\infty} \sum_{j=1}^{\infty}\left(\frac{\lambda_{j}^{\left(c_{k}\right)}}{r}\right)^{c_{k}} S_{c_{k} ; \lambda_{j}^{\left(c_{k}\right)}}(r)\left[\sum_{l=1}^{\gamma_{k}} f_{l}^{(k)}\left(\lambda_{j}^{\left(c_{k}\right)}\right) Y_{k}^{(l)}(\underline{x})\right]
$$

where $c_{k}=\frac{m+2 k-2}{2}, \lambda_{j}^{\left(c_{k}\right)}$ are the scaled positive zeros of the Bessel function $J_{c_{k}}$ given by (1.2), $S_{c_{k} ; \lambda_{j}^{\left(c_{k}\right)}}$ is given by (3.3), and the series (3.18) is convergent in the $L^{2}$-norm sense. In particular,

$$
f^{(N)}(\underline{x})=\sum_{k=0}^{N} \sum_{j=1}^{\infty}\left(\frac{\lambda_{j}^{\left(c_{k}\right)}}{r}\right)^{c_{k}} S_{c_{k} ; \lambda_{j}^{\left(c_{k}\right)}}(r)\left[\sum_{l=1}^{\gamma_{k}} f_{l}^{(k)}\left(\lambda_{j}^{\left(c_{k}\right)}\right) Y_{k}^{(l)}(\underline{x})\right],
$$


converges to $f$ in the $L^{2}$ sense, and for every fixed $N$, the series (3.19) point-wisely, absolutely and uniformly convergent in $\mathbf{R}^{m}$.

Proof. Since $f \in L^{2}\left(\mathbf{R}^{m}\right)$, it follows from (3.1) and the expansion (3.16) that $f$ has the decomposition

$$
f(\underline{x})=\sum_{k=0}^{\infty} f_{k}(\underline{x})=\sum_{k=0}^{\infty} \sum_{l=1}^{\gamma_{k}} f_{l}^{(k)}(r) Y_{k}^{(l)}(\underline{x})=\sum_{k=0}^{\infty} \sum_{l=1}^{\gamma_{k}} f_{l}^{(k)}(r) r^{k} Y_{k}^{(l)}\left(\underline{x}^{\prime}\right),
$$

where $\underline{x}=r \underline{x}^{\prime}, r=|\underline{x}|$ and $\left\{Y_{k}^{(l)}\left(\underline{x}^{\prime}\right)\right\}_{l=1}^{\gamma_{k}}$ is an orthonormal basis of $Y_{k}$. The convergence is in the $L^{2}$ sense.

By taking Fourier transform on both sides of (3.20), we have

$$
\widehat{f}(\underline{t})=\sum_{k=0}^{\infty} \widehat{f_{k}}(\underline{t})=\sum_{k=0}^{\infty} \sum_{l=1}^{\gamma_{k}} \widehat{f_{l}^{(k)}}(s) Y_{k}^{(l)}(\underline{t})=\sum_{k=0}^{\infty} \sum_{l=1}^{\gamma_{k}} \widehat{f_{l}^{(k)}}(s) s^{k} Y_{k}^{(l)}\left(\underline{t}^{\prime}\right) .
$$

Since $f$ is bandlimited with bandwidth $\sigma$, for $s>\sigma,(3.21)$ becomes

$$
0=\sum_{k=0}^{\infty} \sum_{l=1}^{\gamma_{k}} \widehat{f_{l}^{(k)}}(s) s^{k} Y_{k}^{(l)}\left(\underline{t}^{\prime}\right) .
$$

Multiplying the both sides by $Y_{k^{\prime}}^{\left(l^{\prime}\right)}\left(\underline{t}^{\prime}\right)\left(k^{\prime} \in \mathbf{N}\right.$ and $\left.l^{\prime}=1, \cdots, \gamma_{k}\right)$, integrating over the unit sphere $\Sigma_{m-1}$ term-by-term, and using the orthogonality of the spherical harmonics

$$
\int_{\Sigma_{m-1}} Y_{k}^{(l)}\left(\underline{t}^{\prime}\right) \overline{Y_{k^{\prime}}^{\left(l^{\prime}\right)}}\left(\underline{t}^{\prime}\right) d \underline{t}^{\prime}=0 \quad \text { if } l \neq l^{\prime} \text { or } k \neq k^{\prime}
$$

and

$$
\int_{\Sigma_{m-1}}\left|Y_{k}^{(l)}\left(\underline{t}^{\prime}\right)\right|^{2} d \underline{t}^{\prime}=1
$$

we obtain

$$
0=\sum_{k=0}^{\infty} \sum_{l=1}^{\gamma_{k}} \widehat{f_{l}^{(k)}}(s) s^{k} \int_{\Sigma_{m-1}} Y_{k}^{(l)}\left(\underline{t}^{\prime}\right) \overline{Y_{k^{\prime}}^{\left(l^{\prime}\right)}}\left(\underline{t}^{\prime}\right) d \underline{t}^{\prime}=\widehat{f_{l^{\prime}}^{\left(k^{\prime}\right)}}(s) s^{k^{\prime}} .
$$

Therefore, $\widehat{f_{l}^{(k)}}(s)=0$ for $k \in \mathbf{N}, l=1, \ldots, \gamma_{k}$ and $s>\sigma$. This shows that supp $\widehat{f_{l}^{(k)}}(|\cdot|) \subset[0, \sigma], k \in \mathbf{N}$ and $l=1,2, \ldots, \gamma_{k}$ and $f_{l}^{(k)}(r) Y_{k}^{(l)}(\underline{x}) \in L^{2}\left(\mathbf{R}^{m}\right)$ are all bandlimited. Using (3.14) in Theorem 3.2, we get

$$
f_{l}^{(k)}(r) Y_{k}^{(l)}(\underline{x})=\sum_{j=1}^{\infty} f_{l}^{(k)}\left(\lambda_{j}^{\left(c_{k}\right)}\right)\left(\frac{\lambda_{j}^{\left(c_{k}\right)}}{r}\right)^{c_{k}} S_{c_{k} ; \lambda_{j}^{\left(c_{k}\right)}}(r) Y_{k}^{(l)}(\underline{x}) .
$$

Adding up those terms in $k, j, l$ we obtain (3.18). 
The $L^{2}$ convergence of (3.18) is based on the $L^{2}$ convergence of (3.24) and that of (3.17). The point-wise convergence property of (3.19) follows from the corresponding property of the series discussed in Theorem 3.2.

The theorem asserts that by ignoring an error in the $L^{2}$ sense, the principal part (3.19) of the sampling formula (3.18) may be uniformly and absolutely in the point-wise sense approximated by the corresponding interpolation series. A discussion following the idea of (2.6) regarding any reminder of the series is possible but complicated.

\section{Sampling in Relation to Decomposition into Homogeneous Left-Monogenic Functions}

In this section we establish the corresponding sampling results in relation to homogeneous left-monogenic functions that refines what are obtained for homogeneous harmonics in the last section. We first introduce basic concepts in relation to leftmonogenic functions in Clifford analysis ([4] and [10]).

We will be working with $\mathbf{R}_{1}^{m}$, the real-linear span of $\mathbf{e}_{0}, \mathbf{e}_{1}, \cdots, \mathbf{e}_{m}$, where $\mathbf{e}_{0}$ is identical with 1 and $\mathbf{e}_{i} \mathbf{e}_{j}+\mathbf{e}_{j} \mathbf{e}_{i}=-2 \delta_{i j}(i, j=1,2, \ldots, m)$. The real$(m+1)$-dimensional linear space $\mathbf{R}_{1}^{m}$ is embedded into the real-Clifford algebra $\mathbf{R}^{(m)}$ and the complex-Clifford algebra $\mathbf{C}^{(m)}$ generated by $\mathbf{e}_{1}, \cdots, \mathbf{e}_{m}$ over the real and complex number fields, respectively. A typical element in $\mathbf{R}_{1}^{m}$ is denoted by $x=x_{0}+\underline{x}$, where $x_{0} \in \mathbf{R}$ and $\underline{x}=x_{1} \mathbf{e}_{1}+\cdots+x_{m} \mathbf{e}_{m} \in \mathbf{R}^{m}$, the latter being identical with the Euclidean space $\mathbf{R}^{m}$. We usually write $x=r x^{\prime}$, where $r=|x|$. A typical element in the complex-Clifford algebra $\mathbf{C}^{(m)}$ is

$$
x=\sum_{S=\emptyset \text { or }\left(j_{1}, \cdots, j_{l}\right)} x_{S} \mathbf{e}_{S},
$$

where $1 \leq j_{1}<\cdots j_{l} \leq m, 1 \leq l \leq m, x_{S} \in \mathbf{C}, \mathbf{e}_{S}=\mathbf{e}_{j_{1}} \cdots \mathbf{e}_{j_{l}}, \mathbf{e}_{\emptyset}=\mathbf{e}_{0}$. Functions to be studied in this note are $\mathbf{R}_{1}^{m}$-variable and complex-Clifford algebra-valued. A general function is of the form $f(x)=\sum_{S} f_{S}(x) \mathbf{e}_{S}$, and the component functions $f_{S}$ are complex-valued. Left- and right-monogenic functions are introduced via the generalized Cauchy-Riemann operator $D=\frac{\partial}{\partial x_{0}} \mathbf{e}_{0}+\frac{\partial}{\partial x_{1}} \mathbf{e}_{1}+\cdots+\frac{\partial}{\partial x_{m}} \mathbf{e}_{m}: \mathrm{A}$ function $f$ with continuous first order derivatives is said to be left-monogenic, or right-monogenic, if

or

$$
D f=\sum_{i=0}^{m} \sum_{S} \frac{\partial f_{S}}{\partial x_{i}} \mathbf{e}_{i} \mathbf{e}_{S}=0
$$

$$
f D=\sum_{i=0}^{m} \sum_{S} \frac{\partial f_{S}}{\partial x_{i}} \mathbf{e}_{S} \mathbf{e}_{i}=0,
$$

in its domain, respectively. In this note we only study left-monogenic functions. The theory for right-monogenic functions is parallel. Note that there exist Cauchy's 
Theorem and Cauchy's formula in this setting ([4] or [7]). There exists also a Taylor and Laurent series theory for left-monogenic functions. For $m=1$ we have $\mathbf{R}_{1}^{1}=\mathbf{C}=\mathbf{R}^{(1)}$, the space of complex numbers, where left- and right- monogenic functions both coincide with holomorphic functions.

Now we can establish the sampling formula via the decomposition of the spaces of spherical harmonics ([7] p.162) into the spaces of spherical left-monigenics.

For any $k \in \mathbf{N}$, there holds

$$
Y_{k}=M_{k}^{+} \bigoplus M_{k-1}^{-},
$$

where $M_{k}^{+}$and $M_{k}^{-}$are the spaces consisting of the restrictions to the unit sphere $\Sigma_{m-1}$ of, respectively, the functions in $M_{k}^{+}$and $M_{k}^{-}$. The notations $M_{k}^{+}$and $M_{k}^{-}$ are the spaces of left-monogenic homogeneous functions in $\mathbf{R}_{1}^{m}$ of degree $k$ and in $\mathbf{R}_{1}^{m} \backslash\{0\}$ of degree $-(m+k-1)$, respectively. The elements of $M_{k}^{+}$and $M_{k}^{-}$are called spherical monogenics, or surface spherical monogenics, of degree $k$.

For any $f_{k} \in N_{k}, k \in \mathbf{N}$, the space invariant under Fourier transformation discussed in Section 3, combining (3.16) and (4.1), we have

$$
f_{k}(\underline{x})=\sum_{l=1}^{\gamma_{k}} f_{l}^{(k)}(r) r^{k}\left(g_{l}^{+}\left(\underline{x}^{\prime}\right)+g_{l}^{-}\left(\underline{x}^{\prime}\right)\right),
$$

where $r=|\underline{x}|, g_{l}^{+} \in M_{k}^{+}$and $g_{l}^{-} \in M_{k-1}^{-}$. Therefore,

$$
f_{k}(\underline{x})=\sum_{l=1}^{\gamma_{k}} f_{l}^{(k)}(r) P_{l}(\underline{x})+\sum_{l=1}^{\gamma_{k}} h_{l}^{(k)}(r) Q_{l}(\underline{x}),
$$

where $P_{l} \in M_{k}^{+}, Q_{l} \in M_{k-1}^{-}, h_{l}^{(k)}(r)=f_{l}^{(k)}(r) r^{m+2 k-2}$ and $f_{l}^{(k)}$ satisfy

$$
\int_{0}^{\infty}\left|f_{l}^{(k)}(r)\right|^{2} r^{m+2 k-1} d r<\infty,
$$

so $h_{l}^{(k)}(r)=f_{l}^{(k)}(r) r^{m+2 k-2}$ satisfies the inequality

$$
\int_{0}^{\infty}\left|h_{l}^{(k)}(r)\right|^{2} r^{-(m+2 k-3)} d r<\infty .
$$

We have

$$
\begin{aligned}
& N_{0}=\Omega^{0} \\
& N_{k}=\Omega^{k} \bigoplus \Omega^{-k}, k \in \mathbf{N},
\end{aligned}
$$

where $\Omega^{k}, k \geq 0$, is the right-Clifford module of finite linear combinations of functions of the form $f(r) P(\underline{x})$, where $r=|\underline{x}|, P \in M_{k}^{+}$and $f$ is a function satisfying $\int_{0}^{\infty}|f(r)|^{2} r^{m+2 k-1} d r<\infty ; \Omega^{-k}, k>0$, is the right-Clifford module of finite linear combinations of functions of the form $h(r) Q(\underline{x})$, where $r=|\underline{x}|, Q \in M_{k-1}^{-}$and $h$ 
is a function satisfying $\int_{0}^{\infty}|h(r)|^{2} r^{-(m+2 k-3)} d r<\infty$. Note that for $k \in \mathbf{N}$, the space $\Omega^{-k}$ corresponds to the space $M_{k-1}^{-}$.

The direct sum decomposition

$$
L^{2}\left(\mathbf{R}^{m}\right)=\sum_{k=-\infty}^{\infty} \bigoplus \Omega^{k}
$$

holds in the sense that: the subspaces $\Omega^{k}$ are closed and mutually orthogonal, $k \in \mathbf{Z}$. Every function of $L^{2}\left(\mathbf{R}^{m}\right)$ is a limit of finite linear combinations of functions in $\bigcup_{k=-\infty}^{\infty} \Omega^{k}$. Fourier transformation maps each subspace $\Omega^{k}$ into itself. This result is an extension of the classical $m=1$ case ([15] (1.4) Chapter IV) to any $m \in \mathbf{N}([8])$.

For any $g_{k} \in \Omega^{k}, k \in \mathbf{Z}$,

$$
g_{k}(\underline{x})=\sum_{l=1}^{\gamma_{k}} g_{l}^{(k)}(r) R_{k}^{(l)}(\underline{x}),
$$

where $r=|\underline{x}|, R_{k}^{(l)} \in M_{k}^{+}$, if $k \geq 0$; and $R_{k}^{(l)} \in M_{|k|-1}^{-}$, if $k<0$.

In the following, assume the dimension $m \geq 2$, we derive the sampling expansion for bandlimited square integrable functions in relation to the decomposition $(4.3)$.

Theorem 4.1. Let $f \in \Omega^{k}, k \in \mathbf{Z}$, with the form $f(\underline{x}):=g(r) R(\underline{x})(r=|\underline{x}|)$, where $R(\underline{x}) \in M_{k}^{+}$, if $k \geq 0$; and $R(\underline{x}) \in M_{|k|-1}^{-}$, if $k<0$, and $g$ be bandlimited with bandwidth $\sigma$. Then $f$ can be constructed completely via

$$
f(\underline{x})=\sum_{j=1}^{\infty} g\left(\lambda_{j}^{\left(c_{|k|}\right)}\right)\left(\frac{\lambda_{j}^{\left(c_{|k|}\right)}}{r}\right)^{\operatorname{sgn}(k) c_{|k|}} S_{c_{|k| ; \lambda_{j}}^{\left(c_{|k|}\right)}}(r) R(\underline{x})
$$

where $\lambda_{j}^{\left(c_{|k|}\right)}$ are the scaled positive zeros of the Bessel function $J_{c_{|k|}}$ given by (1.2), $S_{c_{|k|} ; \lambda_{j}^{\left(c_{|k|}\right)}}$ is given by (3.3), $c_{|k|}=\frac{m+2|k|-2}{2}(k \in \mathbf{Z})$, sgn $(k)$ is the signum function that takes the value $+1,-1$ or 0 , respectively, for $k>0, k<0$ or $k=0$ and the series (4.5) being convergent in $L^{2}$-norm, absolutely and uniformly convergent point-wisely on whole space $\mathbf{R}^{m}$.

Proof. The Fourier transform of the function $f(\underline{x}):=g(r) R(\underline{x})(r=|\underline{x}|)$ where $R(\underline{x}) \in M_{k}^{+}$, if $k \geq 0$; and $R(\underline{x}) \in M_{|k|-1}^{-}$, if $k<0$, with an abuse of notation but informatively, can be expressed as

$$
\widehat{f}(\underline{t})=\widehat{g}(s) R(\underline{t}) \quad(s=|\underline{t}|)
$$


and

$$
\widehat{g}(s)=i^{-k}(2 \pi)^{\frac{m}{2}} \int_{0}^{\infty}\left[g(r) r^{\operatorname{sgn}(k) c_{|k|}}\right]\left[s^{-\operatorname{sgn}(k) c_{|k|}} J_{c_{|k|}}(s r)\right] r d r,
$$

where $c_{|k|}=\frac{m+2|k|-2}{2}(k \in \mathbf{Z})$ and $\operatorname{sgn}(k)$ is the signum function that takes the value $+1,-1$ or 0 for $k>0, k<0$ or $k=0$ ([8] Theorem 2$)$.

By the assumption supp $\widehat{g} \subset[0, \sigma]$ and $g \in L^{2}([0, \sigma])$, the radial function $g$ can be obtained via the integral formula

$$
g(r)=i^{-k}(2 \pi)^{-\frac{m}{2}} \int_{0}^{\sigma}\left[\widehat{g}(s) s^{\operatorname{sgn}(k) c_{|k|}}\right]\left[r^{-\operatorname{sgn}(k) c_{|k|}} J_{c_{|k|}}(r s)\right] s d s .
$$

The linear integral transformation (4.6) maps $F(s)=i^{-k}(2 \pi)^{-\frac{m}{2}} \widehat{g}(s) s^{\operatorname{sgn}(k) c_{|k|}}$ to $g(r)$, with weight function $\rho(s)=s$, interval $I=[0, \sigma]$ and kernel $K(s, r)=$

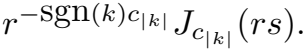

Let $r_{j}=\lambda_{j}^{\left(c_{|k|}\right)}$ such that $K\left(s, r_{j}\right)=a_{j} \overline{\phi_{j}}(s)$, where

$$
a_{j}=\frac{\sigma J_{c_{|k|}+1}\left(u_{j}^{\left(c_{|k|}\right)}\right)}{\sqrt{2}\left(\lambda_{j}^{\left(c_{|k|}\right)}\right)^{\operatorname{sgn}(k) c_{|k|}}}
$$

and

$$
\overline{\phi_{j}}(s)=\frac{\sqrt{2} J_{c_{|k|}}\left(s \lambda_{j}^{\left(c_{|k|}\right)}\right)}{\sigma J_{c_{|k|}+1}\left(u_{j}^{\left(c_{|k|}\right)}\right)}
$$

forms a complete orthonomal basis in $L^{2}(I, \rho)$. When expand $K(s, r)$ into the series of $\phi_{j}$, the corresponding coefficient

$$
S_{j}(r)=\frac{\sigma J_{c_{|k|}+1}\left(u_{j}^{\left(c_{j}\right)}\right)}{\sqrt{2} r^{\operatorname{sgn}(k) c_{|k|}}} S_{c_{|k|} ; \lambda_{j}^{\left(c_{|k|}\right)}}(r) .
$$

Therefore by the general theory described in Section 2, we have

$$
g(r)=\sum_{j=1}^{\infty} g\left(\lambda_{j}^{\left(c_{|k|}\right)}\right)\left(\frac{\lambda_{j}^{\left(c_{|k|}\right)}}{r}\right)^{\operatorname{sgn}(k) c_{|k|}} S_{c_{|k| ;} ; \lambda_{j}^{\left(c_{|k|}\right)}}(r)
$$

where $r=|\underline{x}|$. The desired relation (4.5) follows. The $L^{2}$ and point-wise convergence properties may be proved by using the same methods as in Theorem 3.2.

Theorem 4.2. If $f \in L^{2}\left(\mathbf{R}^{m}\right)$ and $f$ is bandlimited with bandwidth $\sigma$, then $f$ can be written as

$$
f(\underline{x})=\sum_{k=-\infty}^{\infty} g_{k}(\underline{x})=\sum_{k=-\infty}^{\infty} \sum_{l=1}^{\gamma_{k}} g_{l}^{(k)}(r) R_{k}^{(l)}(\underline{x})
$$


where $R_{k}^{(l)} \in M_{k}^{+}$, if $k \geq 0$; and $R_{k}^{(l)} \in M_{|k|-1}^{-}$, if $k<0$. Moreover, $f$ can be constructed completely via

$$
f(\underline{x})=\sum_{k=-\infty}^{\infty} \sum_{l=1}^{\gamma_{k}} \sum_{j=1}^{\infty} g_{l}^{(k)}\left(\lambda_{j}^{\left(c_{|k|}\right)}\right)\left(\frac{\lambda_{j}^{\left(c_{|k|}\right)}}{r}\right)^{\operatorname{sgn}(k) c_{|k|}} S_{c_{|k|} ; \lambda_{j}^{\left(c_{|k|}\right)}(r)} R_{k}^{(l)}(\underline{x}),
$$

where $\lambda_{j}^{\left(c_{|k|}\right)}$ are the scaled positive zeros of the Bessel function $J_{c_{|k|}}$ given by (1.2), $S_{c_{|k|} ; \lambda_{j}^{\left(c_{|k|}\right)}}$ is given by (3.3), $c_{|k|}=\frac{m+2|k|-2}{2}(k \in \mathbf{Z})$, sgn $(k)$ is the signum function that takes the value $+1,-1$ or 0, for $k>0, k<0$ or $k=0$. The series (4.8) converges to $f$ in the $L^{2}$ sense. In particular,

$f^{(N, M)}(\underline{x})=\sum_{k=-M}^{N} \sum_{l=1}^{\gamma_{k}} \sum_{j=1}^{\infty} g_{l}^{(k)}\left(\lambda_{j}^{\left(c_{|k|}\right)}\right)\left(\frac{\lambda_{j}^{\left(c_{|k|}\right)}}{r}\right)^{s g n(k) c_{|k|}} S_{\left.c_{|k|} ; \lambda_{j}^{(c|k|}\right)}(r) R_{k}^{(l)}(\underline{x}),(4.9)$

converges to $f$ in the $L^{2}$ sense as $N, M \rightarrow \infty$, and for every fixed pair of positive integers $N, M$ the series (4.9) point-wisely, absolutely and uniformly convergent in $\mathbf{R}^{m}$.

Proof. As consequence of (4.3) and (4.4), $f$ can be written as

$$
f(\underline{x})=\sum_{k=-\infty}^{\infty} g_{k}(\underline{x})=\sum_{k=-\infty}^{\infty} \sum_{l=1}^{\gamma_{k}} g_{l}^{(k)}(r) R_{k}^{(l)}(\underline{x}) \quad(r=|\underline{x}|),
$$

where $R_{k}^{(l)} \in M_{k}^{+}$, if $k \geq 0$; and $R_{k}^{(l)} \in M_{|k|-1}^{-}$, if $k<0$. The convergence is in the $L^{2}$ sense.

Applying Fourier transform on both sides of (4.10), we have

$$
\widehat{f}(\underline{t})=\sum_{k=-\infty}^{\infty} \widehat{g_{k}}(\underline{t})=\sum_{k=-\infty}^{\infty} \sum_{l=1}^{\gamma_{k}} \widehat{g_{l}^{(k)}}(s) R_{k}^{(l)}(\underline{t}) \quad(s=|\underline{t}|) .
$$

Since $\widehat{f}$ vanishes outsides $B(0, \sigma)$, for any $s>\sigma,(4.11)$ becomes

$$
0=\sum_{k=-\infty}^{\infty} \sum_{l=1}^{\gamma_{k}} \widehat{g_{l}^{(k)}}(s) R_{k}^{(l)}(\underline{t})
$$

Owing to the orthogonality property of $R_{k}^{(l)}$, the same reasoning as proving Theorem 3.3 gives that supp $\widehat{g_{l}^{(k)}} \subset[0, \sigma]\left(k=\ldots,-1,0,1, \ldots, l=1,2, \ldots, \gamma_{k}\right)$. That is, $g_{l}^{(k)}$ is bandlimited with bandwidth $\sigma$. Since $g_{l}^{(k)}(r) R_{k}^{(l)}(\underline{x}) \in L^{2}\left(\mathbf{R}^{m}\right)$, applying Theorem 4.1, relation (4.5) gives

$$
g_{l}^{(k)}(r) R_{k}^{(l)}(\underline{x})=\sum_{j=1}^{\infty} g_{l}^{(k)}\left(\lambda_{j}^{\left(c_{|k|}\right)}\right)\left(\frac{\lambda_{j}^{\left(c_{|k|}\right)}}{r}\right)^{\operatorname{sgn}(k) c_{|k|}} S_{c_{|k|} ; \lambda_{j}^{\left(c_{|k|}\right)}}(r) R_{k}^{(l)}(\underline{x}),
$$


where $S_{c_{|k|} ; \lambda_{j}^{\left(c_{|k|}\right)}}$ is given by $(3.3), c_{|k|}=\frac{m+2|k|-2}{2}(k \in \mathbf{Z})$ and $\lambda_{j}^{\left(c_{|k|}\right)}$ are the scaled positive zeros of the Bessel function $J_{c_{|k|}}$ given by (1.2). Substitute (4.13) into the right hand side of (4.10). We get the desired expansion (4.8). The convergence properties may be proved similarly as in the proof of Theorem 3.3.

\section{References}

[1] G. Abramowitz and I. Stegun, Handbook of Mathematical Functions, Dover, New York, 1972.

[2] George B. Arfken and Hans J. Weber, Mathematical Methods for Physicists, Harcourt, San Diego, 2001.

[3] F. Bowman, Introduction to Bessel Functions, New York: Dover, 1958.

[4] F. Brackx, R. Delanghe and F. Sommen, Clifford Analysis, Research Notes in Mathematics, Vol. 76, Pitman Advanced Publishing Company, Boston, London, Melbourne, 1982.

[5] P. L. Butzer, A survey of the Whittaker-Shannon sampling theorem and some of its extensions, J. Math. Res. Expos., 3 (1983), 185-212.

[6] P. L. Butzer and G. Nasri-Roudsari, Kramer's sampling theorem in signal analysis and its role in mathematics, in Sampling Theorey and Signal Analysis II, J. R. Higgins and R. Stens, eds., Oxford University Press, Oxford, UK, 1999, 96-129.

[7] R. Delanghe, F. Sommen and V. Soucek, Clifford algebra and spinor valued functions, A function theory for Dirac operator. Kluwer, Dordrecht, 1992.

[8] M. Fei and T. Qian, Direct sum decomposition of $L^{2}\left(\mathbf{R}_{1}^{m}\right)$ into subspaces invariant under Fourier transformation, J. Fourier Anal. Appl., 12(2), (2006), 145-155.

[9] A. G. Garcia, Orthogonal Sampling Formulas: A Unified Approach, SIAM Review Vol. $42(3),(2000), 499-512$.

[10] J. E. Gilbert and M. A. M. Murray, Clifford Algebras and Dirac operators in Harmonic Analysis, Cambridge Studies in Advanced Mathematics, 26, Cambridge University Press, 1991.

[11] G. H. Hardy, Notes on special systems of orthogonal functions, IV: The Whittaker's cardinal series, Proc. Camb. Phil. Soc., 37 (1941), 331-348.

[12] J. R. Higgins, Five short stories about the cardinal series, Bull. Amer. Math. Soc., 12 (1985), 45-89.

[13] A. J. Jerri, The Shannon sampling theorem-Its various extensions and applications: A tutorial review, Proc. IEEE, 65 (1977), 1565-1596.

[14] N.N. Lebedev, Special Functions and Their Applications, Prentice-Hall, London, 1965.

[15] E. Stein and G. Weiss, Introduction to Fourier analysis on Euclidean spaces, Princeton university press, 1971.

[16] G. N. Watson, A Treatise on the Theory of Bessel Functions, Second Edition, Cambridge University Press, 1966. 
K.I. Kou

Faculty of Science and Technology

University of Macau

Av. Padre Tomas Pereira, S. J., Taipa, Macao

e-mail: kikou@umac.mo

T. Qian

Faculty of Science and Technology

University of Macau

Av. Padre Tomas Pereira, S. J., Taipa, Macao

e-mail: fsttq@umac.mo

F. Sommen

Department of Mathematical Analysis

University of Ghent

Galglaan 2, B-9000 Gent, Belgium

e-mail: fs@cage.rug.ac.be 